\title{
Late Reperfusion of A Totally Occluded Infarct- Related Artery Increases Granulocyte-Colony Stimulation Factor and Reduces Stroma-Derived Factor-1 $\alpha$ Blood Levels in Patients With Ongoing Ischemia After Acute Myocardial Infarction
}

\author{
Li-Tang Kuo, ${ }^{1}$ MD, Shih-Jen Chen, ${ }^{1}$ MD, Wen-Jin Cherng, ${ }^{1}$ MD, \\ Ning-I YAng, ${ }^{1}$ MD, Chen-Chin LeE, ${ }^{1}$ MD, Chi-Wen Cheng, ${ }^{1}$ MD, \\ Subodh Verma, ${ }^{2} \mathrm{MD}$, and Chao-Hung WANG, ${ }^{1} \mathrm{MD}$
}

\begin{abstract}
SUMMARY
After acute myocardial infarction (AMI), reopening of a totally occluded infarct-related artery (IRA) at a subacute stage is still controversial in symptom-free patients. However, in patients with persistent ischemic symptoms and inadequate collaterals to the infarct area, recanalization is thought to provide beneficial effects. In addition to augmenting myocardial perfusion, we hypothesized that the benefit of recanalization involves the manipulation of circulating stem cell-mobilizing cytokines. This study included 30 patients with a totally occluded IRA and ongoing ischemic symptoms (the study group) and 30 patients with a partially occluded IRA (the control group). All patients underwent successful angioplasty and/or stenting. Before and immediately after the coronary intervention, blood granulocyte-colony-stimulating factor (G-CSF), stem-cell factor (SCF), vascular endothelial growth factor (VEGF), and stroma-derived factor-1 (SDF-1 $\alpha$ ) were measured. After recanalization, G-CSF levels significantly increased in the study group compared to the control group $(P=0.03)$. SDF- $1 \alpha$ levels in the study group decreased relative to the controls $(P=0.02)$. However, no significant changes in VEGF or SCF levels between the two groups were found. In the multivariate analysis, reopening of a totally occluded IRA was independently and significantly associated with changes in G-CSF and SDF- $1 \alpha$ levels after recanalization. In conclusion, our data suggest that the benefits of late reperfusion of a totally occluded IRA in patients with ongoing myocardial ischemia may involve mechanisms associated with stem cell-mobilizing and plaque-stabilizing cytokines. This study provides the rationale to investigate serial changes in cytokines and the numbers of circulating progenitors after reperfusion in the future. (Int Heart J 2009; 50: 433-444)
\end{abstract}

Key words: Myocardial infarction, Cytokine, Reperfusion

From the ${ }^{1}$ Division of Cardiology, Department of Internal Medicine, Chang Gung Memorial Hospital, Keelung, Chang Gung University College of Medicine, Taoyuan, Taiwan. ${ }^{2}$ Division of Cardiac Surgery, Toronto General Hospital, and Division of Cardiac Surgery, St. Michael's Hospital, Toronto, Canada.

Address for correspondence: Chao-Hung Wang, MD, Division of Cardiology, Department of Internal Medicine, Chang Gung Memorial Hospital, 222 Mai Chin Road, Keelung, Taiwan.

Received for publication November 26, 2008.

Revised and accepted March 30, 2009. 
THE Open Artery Trial (TOAT study) and recent large-scale studies have suggested that recanalization of occluded infarct-related arteries (IRAs) has an adverse effect on remodeling in symptom-free patients approximately 3 days to 1 month after an acute myocardial infarction (AMI), probably due to the trend toward excess reinfarction during 4 years follow-up. ${ }^{1,2)}$ However, reopening the totally occluded IRA tends to increase exercise tolerance and improves the quality of life. Furthermore, in the Total Occlusion Study of Canada (TOSCA)-2 trial, the recanalization group had a trend toward a greater improvement in target-region wall motion. ${ }^{3)}$ The absolute effect of recanalization was greatest in patients with a low left ventricular ejection fraction who actually were at greatest risk for left ventricular dilation. Although the debate still exists in symptomfree patients, it is currently suggested that recanalization of totally occluded IRAs provides beneficial effects in patients with persistent ischemic symptoms or with inadequate collaterals to the infarct area.

Recently, data from animal and clinical studies have supported bone marrow stem cell therapy as having beneficial effects on cardiac functional improvement after an AMI. ${ }^{4-8)}$ Cytokine-induced mobilization of stem cells from the bone marrow is a promising alternative..$^{9-11)}$ Previous studies showed that administration of granulocyte-colony stimulation factor (G-CSF) and stem-cell factor (SCF) after an MI reduces myocardial damage and cardiac mortality. ${ }^{8)}$ Vascular endothelial growth factor (VEGF) and stroma-derived factor-1 $\alpha$ (SDF- $1 \alpha)$ are also potent mobilizers of bone marrow stem/progenitor cells. ${ }^{12-15)}$ Recent studies have revealed that after an AMI, the damaged cardiac tissues synthesize and secrete all 4 of these cytokines. ${ }^{12,14,16-19)}$ However, a totally occluded IRA with inadequate collateral formation is usually associated with poor or no blood supply to the infarct region, leading to an inadequate flushing-out of the cytokines produced locally and is an obstacle to stem cell homing to injured sites. This study hypothesized that reopening the totally occluded IRA in patients with ongoing myocardial ischemia and poorly perfused infarct areas may increase the blood concentrations of a few stem cell-mobilizing cytokines. The results of this study would provide the rationale to further investigate in the future serial changes in cytokines and the numbers of circulating stem/progenitor cells after reperfusion.

\section{Methods}

Patients: Between November 2004 and June 2006, we consecutively recruited patients with an AMI in whom successful late ( $>$ one day, $<7$ days after the onset of chest pain) percutaneous coronary angioplasty with or without stenting was performed. The diagnosis of an AMI was based on typical chest pain lasting $>30$ minutes, accompanied by elevation of the ST-segment of $\geq 0.1 \mathrm{mV}$ in $\geq 2$ 
adjacent electrocardiographic leads and an increase in the creatine phosphokinase concentration to twice the upper normal limit with a concomitant increase in the creatine kinase-MB isoenzyme. Thrombolytic therapy was given to patients who were sent to the hospital within 12 hours of the onset of chest pain. No primary angioplasty was performed. Patients were separated into a study group and control group. In the study group, patients were enrolled if they had ongoing ischemic symptoms. The IRA was totally occluded with TIMI 0 antegrade flow and collateral circulation of $\leq$ grade I. In the control group, patients had a partially occluded IRA with TIMI flow $\geq$ II. All IRAs achieved TIMI III flow after the coronary intervention. Only 30 patients were recruited in each group. Blood samples were collected before and immediately after the coronary intervention procedures. Informed consent was obtained from all patients: the study protocol was approved by the local Ethics Committee of the Chang Gung Memorial Hospital.

Cardiac catheterization: Cardiac catheterization was performed after obtaining informed consent. Coronary angioplasty and stent implantation were performed using standard percutaneous techniques via a radial or femoral artery approach. Pretreatment with an aspirin/clopidogrel regimen was administered at least 1 day before the coronary intervention. A bolus of 7,000 15,000 units of heparin was given to maintain an activated clotting time of $>300$ seconds. The intervention was performed using nominal-pressure semicompliance balloon dilatation to achieve $<40 \%$ residual stenosis. In case of residual stenosis of $\geq 40 \%$ or the occurrence of a subocclusive dissection, a stent was implanted. Adjunctive highpressure noncompliance balloon dilatation was applied after stent deployment to achieve full stent expansion.

Measurement of blood cytokine levels: Arterial blood was collected from the catheterization sheath 5 minutes before and immediately after the coronary intervention (by the same route on both occasions). Blood samples were immediately placed in tubes with EDTA as an anticoagulant, and then centrifuged for 15 minutes at $4000 \mathrm{rpm}$. Plasma was separated into aliquots and stored at $-80^{\circ} \mathrm{C}$ until used. The plasma levels of G-CSF, SCF, VEGF, and SDF- $1 \alpha$ were quantified by enzyme-linked immunosorbent assays (ELISAs) (Quantikine Immunoassay, R\&D System, MN, USA).

Statistical analysis: Data are expressed as the mean \pm standard error of the mean (SEM). Continuous variables were checked for a normal distribution with the Kolmogorov-Smirnov test. In cases of a nonnormal distribution, nonparametric tests were used (Mann-Whitney $U$-test or Kruskal-Wallis analysis of variance (ANOVA) on ranks). Categorical variables were compared by means of the $\chi^{2}$ test and Fisher's exact test. Interactions among risk factors, clinical variables, angiographic parameters, and the percentage changes in cytokines from the 
baseline to after the coronary intervention were examined by a multivariate analysis using a multiple stepwise linear regression model. Statistical significance was assumed if the null hypothesis could be rejected at $P<0.05$. All statistical analyses were performed using SPSS for Windows version 12.0.

\section{Results}

Baseline characteristics: The coronary intervention was performed on average 4.0 \pm 0.4 days and $3.8 \pm 0.4$ days after the AMI in the control and the study groups, respectively $(P=0.72)$. Compared to the control group, the study group had a lower rate of male gender and number of diseased coronary arteries, a lower incidence of IRAs of the left circumflex coronary artery, and fewer smokers and beta-blocker users (Table I). There was only a slightly lower incidence of using

Table I. Clinical Characteristics of the Study Population

\begin{tabular}{lccc}
\hline & $\begin{array}{c}\text { Study group } \\
(n=30)\end{array}$ & $\begin{array}{c}\text { Control group } \\
(n=30)\end{array}$ & $P$ \\
\hline Age (years) & $63 \pm 2$ & $62 \pm 3$ & 0.763 \\
Male (\%) & $20(67)$ & $29(97)$ & 0.003 \\
$t$-PA (\%) & $4(13)$ & $10(34)$ & 0.125 \\
Killip & $1.7 \pm 0.2$ & $1.6 \pm 0.2$ & 0.633 \\
Previous MI (\%) & $6(20)$ & $7(23)$ & 0.759 \\
LVEF (\%) & $46 \pm 2.8$ & $45 \pm 2.9$ & 0.661 \\
Diseased vessel number & $1.8 \pm 0.2$ & $2.3 \pm 0.2$ & 0.043 \\
Infarct-related artery & & & \\
$\quad$ LAD & $17(57)$ & $14(47)$ & 0.61 \\
$\quad$ LCX & $2(7)$ & $9(30)$ & 0.04 \\
$\quad$ RCA & $11(36)$ & $7(23)$ & 0.39 \\
Stenting for IRA & $14(46)$ & $15(50)$ & 0.98 \\
Risk factors & & & \\
Diabetes mellitus $(\%)$ & $14(47)$ & $11(37)$ & 0.441 \\
Hypertension (\%) & $16(53)$ & $21(70)$ & 0.194 \\
Hyperlipidemia (\%) & $17(57)$ & $19(63)$ & 0.605 \\
$\quad$ Smoking (\%) & $13(43)$ & $23(76)$ & 0.008 \\
Medication & & & \\
Aspirin (\%) & $30(100)$ & $30(100)$ & 0.398 \\
Beta-blocker $(\%)$ & $9(30)$ & $18(60)$ & 0.019 \\
ACE inhibitor $(\%)$ & $16(53)$ & $20(67)$ & 0.303 \\
Clopidogrel (\%) & $30(100)$ & $30(100)$ & 0.647 \\
Nitrate (\%) & $26(87)$ & $29(97)$ & 0.167 \\
Statin (\%) & $7(23)$ & $11(37)$ & 0.267 \\
\hline Data are expressed & & & \\
\hline
\end{tabular}

Data are expressed as the mean \pm SEM. The study group indicates patients with a totally occluded infarct-related coronary artery. The control group indicates patients with a partially occluded artery with TIMI flow $\geq$ II. IRA indicates infarct-related artery; MI, myocardial infarction; $t$-PA, tissue-plasminogen activator; LAD, left anterior descending; LCX, left circumflex; LVEF, left ventricular ejection fraction; ACE, angiotensin-converting enzyme; and RCA, right coronary artery. 
thrombolytic agents in the study group compared to the control group. There were no significant differences between these 2 groups regarding the left ventricular ejection fraction or the Killip class.

Cytokines at the baseline and after reperfusion: There were no significant differences between the 2 groups in terms of all 4 cytokines at the baseline before the coronary intervention, although there was a trend for higher baseline G-CSF, VEGF, and SDF-1 $\alpha$ levels in the study group compared to the control group (Figure A-D). In the control group, there was a significant decrease in the SCF level $(P<0.0001$, Figure $C)$ immediately after the coronary intervention compared to the baseline level (before the intervention); however, there were no significant changes in G-CSF, SDF- $1 \alpha$, or VEGF levels. In terms of changes between before and after the coronary intervention, the study group had a significantly higher elevation in the G-CSF level $(P=0.03$, Figure A), but had a significant decrease in the SDF- $1 \alpha$ level $(P=0.02$, Figure $\mathrm{B})$ compared to the control group. Changes in the SCF and VEGF concentrations were not significant between the 2 groups (Figure C, D).

Parameters correlated with baseline cytokine levels: In the univariate analysis, correlations between the blood concentrations of these 4 cytokines and the clinical parameters are shown in Table II. The multivariate analysis showed that a

A

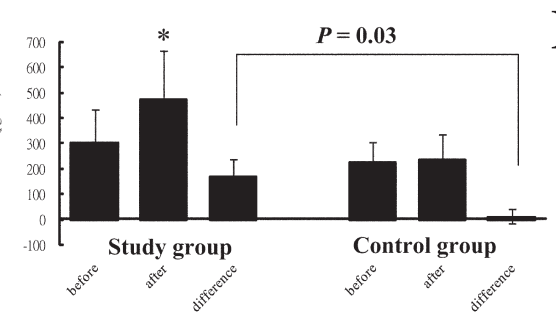

C

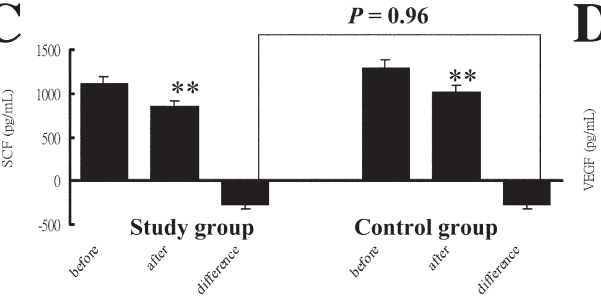

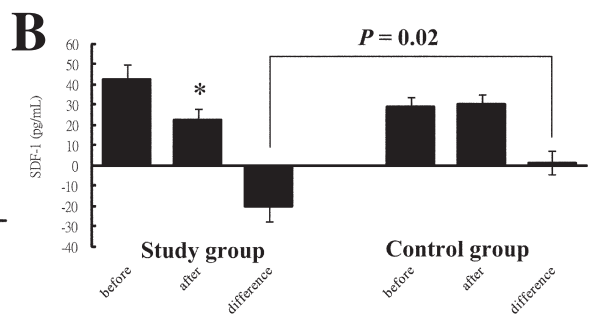

D

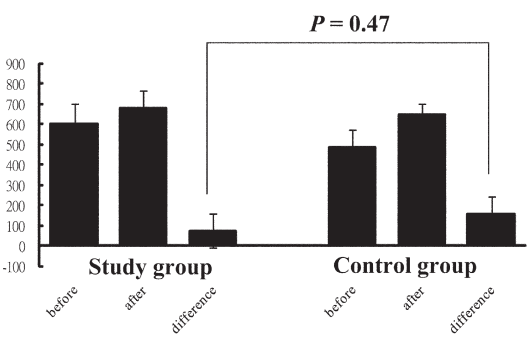

Figure. A-D: Blood levels of cytokines shown before and after the coronary intervention, and their differences. The study group indicates patients with a totally occluded infarct-related coronary artery. The control group indicates patients with a partially occluded artery with TIMI flow $\geq$ II. G-CSF indicates granulocyte-colony stimulation factor; SCF, stem cell factor; VEGF, vascular endothelial growth factor; and SDF- $1 \alpha$, stroma-derived factor- $1 \alpha$. $* P<0.05$, ** $P<0.005$, compared to the baseline (before) cytokine levels. 


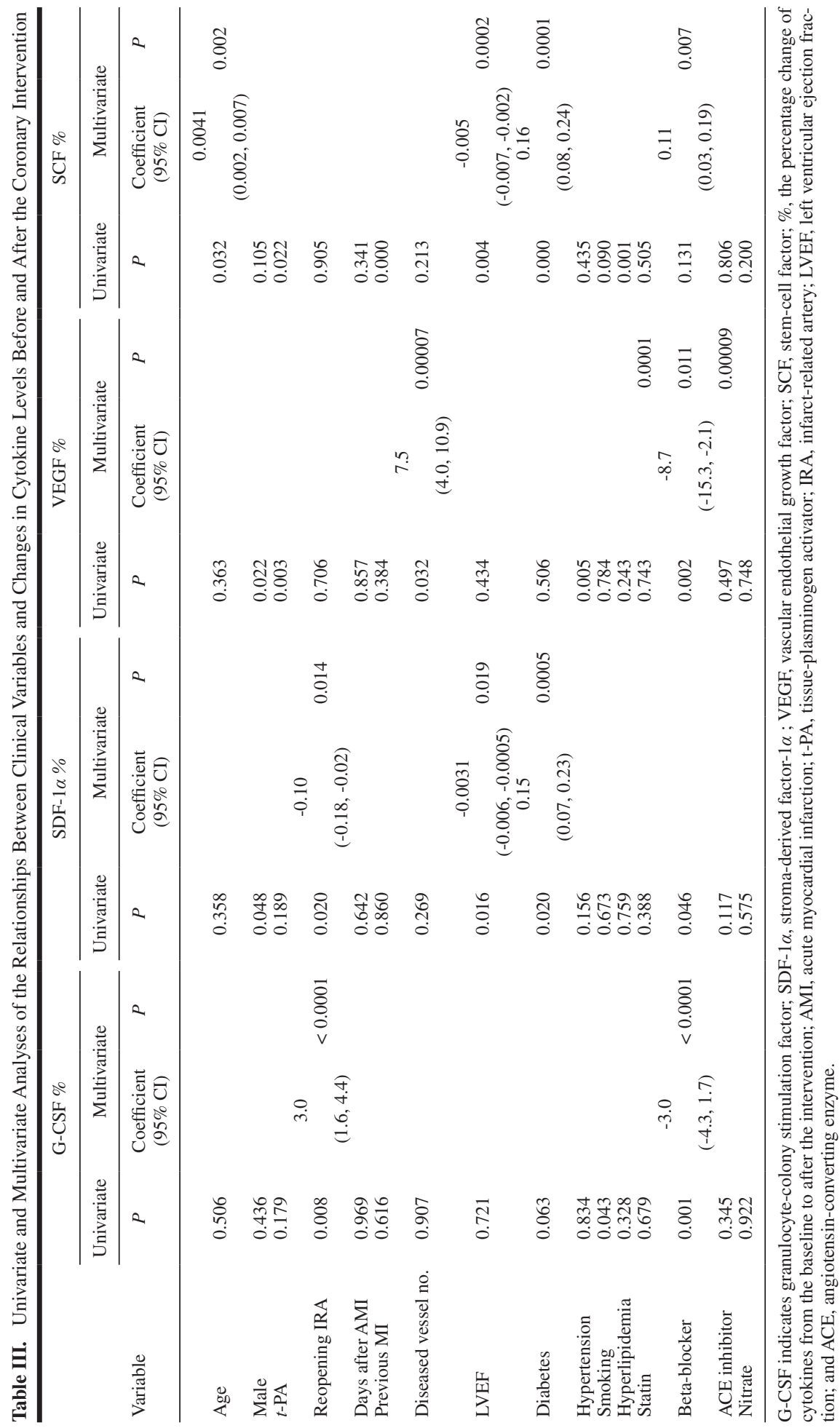


higher baseline G-CSF level was associated with the presence of diabetes and hyperlipidemia. A higher baseline SDF-1 $\alpha$ level was correlated with being female and the use of beta-blockers. The baseline VEGF level was positively correlated with the use of beta-blockers, but negatively correlated with smoking and the presence of hypertension. A higher baseline SCF level was associated with the use of beta-blockers and a higher left ventricular ejection fraction.

Factors related to changes in cytokines from the baseline to post-reperfusion: In the univariate analysis, Table III shows the correlations between changes in these 4 cytokine blood levels and the clinical parameters. In the multivariate analysis, the increase in the G-CSF level was positively correlated with reopening of the IRA, but negatively with the use of beta-blockers. The increase in the SDF-1 $\alpha$ level was positively associated with the presence of diabetes mellitus, but negatively with reopening of the IRA and the left ventricular ejection fraction. The increase in the VEGF level was positively correlated with the number of diseased coronary arteries, and the use of statins, but negatively correlated with the use of beta-blockers and ACE inhibitors. The increase in the SCF level was positively associated with age, the presence of diabetes, and the use of betablockers, but negatively associated with left ventricular ejection fraction.

\section{Discussion}

After an AMI, reopening the totally occluded IRA at the subacute stage is still controversial in symptom-free patients. However, in patients with persistent ischemic symptoms and inadequate collaterals to the infarct area, recanalization of the occluded IRA is thought to provide beneficial effects. Furthermore, previous studies demonstrated that reopening the totally occluded IRA tends to increase exercise tolerance and improves the quality of life. ${ }^{2)}$ In addition to improving antegrade myocardial perfusion, our study provides other associated mechanisms involving the manipulation of circulating stem cell-mobilizing and plaque-stabilizing cytokines.

G-CSF: G-CSF is a well-known potent mobilizer of stem/progenitor cells into the peripheral blood and is currently widely used for transplantation of hematopoietic progenitor cells instead of whole bone marrow in current hematological practice. Pharmacological administration of G-CSF has been used as a noninvasive option for regeneration of myocardial tissue in patients with congestive heart failure or after an AMI. ${ }^{20)}$ Our study suggested that opening an occluded vessel might help stem-cell homing to the injured sites as well as facilitate cell mobilization from the bone marrow by a significant increase in G-CSF levels immediately after the IRA is reperfused. Reopening the IRA may wash out a significant amount of G-CSF which has accumulated in the ischemic myo- 
cardium. Previous reports showed that blood G-CSF levels increased after an AMI. $^{21,22)}$ The G-CSF levels in peripheral blood were significantly correlated with circulating $\mathrm{CD} 34^{+}$angiogenic stem cell amounts. ${ }^{21)}$ Furthermore, G-CSF not only accelerates stem-cell mobilization but also has roles in protecting cells from injury and facilitating re-endothelialization in injured vessels. ${ }^{23)} \mathrm{G}-\mathrm{CSF}$ can be synthesized and secreted by ischemic tissues early after an ischemia insult. ${ }^{24)}$ However, recent data suggested that augmentation of vascular progenitor cells in the circulation fails to revascularize the ischemic myocardium unless accompanied by enhanced expression of homing ligands, such as VEGF-2 and SDF-1 $\alpha^{21}$ ) SDF-1 $\alpha$ : SDF-1 $\alpha$ plays a crucial role in postinfarction stem-cell mobilization and engraftment to the ischemic myocardium. ${ }^{14,25)}$ Similar to G-CSF, transcription of SDF-1 $\alpha$ mRNA is transiently upregulated early after an MI or under hypoxic conditions. ${ }^{21,26,27)}$ However, our study showed that the change in SDF$1 \alpha$ levels was in contrast to the change in G-CSF levels in a setting of AMI. Immediately after a totally occluded IRA was reperfused, SDF- $1 \alpha$ levels decreased relative to the controls and baseline.

Previous reports have showed that SDF- $1 \alpha$ levels are lower in patients with acute coronary syndromes, compared to normal controls. ${ }^{28)} \mathrm{SDF}-1 \alpha$ has antiinflammatory and plaque-stabilizing effects in unstable angina. Zernecke, et al and others demonstrated that SDF- $1 \alpha$ can bind with high affinity to cell-surface proteoglycans and activated platelets, which are rich at sites of arterial injury, and in ruptured or angioplastic plaques. ${ }^{18,29)}$ Thus, a substantial proportion of circulating SDF-1 $\alpha$ may be sequestered within the vessel wall in patients with unstable angina or an AMI, particularly in connection with plaque rupture. These mechanisms may explain why the SDF- $1 \alpha$ level became lower immediately after the occluded IRA was opened.

High concentrations of SDF- $1 \alpha$ in the microenvironment within the plaques after reperfusion probably also play a pivotal role in the homing process for stem/progenitor cells. As suggested by the lack of a correlation between CD $34^{+}$cell and SDF- $1 \alpha$ levels, SDF- $1 \alpha$ seems to be a prevalent local mediator for engraftment of stem/progenitor cells rather than a systemic one. ${ }^{21)}$ Other systemic effectors, such as G-CSF, are needed to mobilize stem/progenitor cells towards the ischemic myocardium. Collectively, these data underline the pivotal role of the interplay between the systemic mobilizing effect of G-CSF and the local contribution to engraftment by SDF-1 $\alpha .^{21)}$

VEGF and SCF: In the current study, there were no significant differences in changes of VEGF and SCF levels between the groups. Previously, we showed that tissue VEGF expression peaked approximately 24 hours after ischemic stress. ${ }^{30)}$ At the subacute stage after an AMI, there might not be much VEGF accumulating inside the infarct area. In addition, VEGF is also expressed by 
activated smooth muscle cells and macrophages, ${ }^{31,32)}$ the numbers of which may increase in response to a coronary intervention to similar extents in both groups. The SCF receptor, named c-kit, is present in the circulation and is expressed by endothelial cells. ${ }^{33)}$ Coronary intervention procedures may cause a significant amount of c-kit to shed from vascular endothelial cells. The binding of SCF to circulating c-kit may have interfered with the measurement of SCF levels in our study and might also explain why the levels of SCF decreased immediately after angioplasty and exhibited no significant changes between the groups. Similar to SDF- $1 \alpha$, SCF also binds to activated platelets. ${ }^{34)}$ However, different from SDF$1 \alpha$, SCF levels significantly decreased not only in the study group but also in the control group. This difference might be due to various affinities of SDF- $1 \alpha$ and SCF to activated platelets or injured vascular surface and still needs to be elucidated.

Factors related to cytokine levels: Our data revealed that the use of beta-blockers was associated with higher blood SDF- $1 \alpha$, VEGF, and SCF concentrations at the baseline. Although the associated mechanism is still to be elucidated, these findings suggest that a tolerance to beta-blockers indicates a substantial area of preserved left ventricular function, which may contribute to a significant amount of cytokine synthesis and secretion. On the other hand, it is interesting to investigate whether the use of beta-blockers in a setting of an AMI has direct effects on mobilizing stem/progenitor cells by increasing these cytokines. Regarding changes in cytokines after a coronary intervention, our multivariate analysis demonstrated that reopening an occluded IRA was the only parameter independently associated with changes in both G-CSF and SDF- $1 \alpha$ levels. These relations support the "open-artery hypothesis" even with late reperfusion.

Limitations: In this study, there are a few limitations. First, although patients were continuously recruited, patient recruitment was neither randomized nor blinded. The data might not represent a normal distribution of the AMI population. However, our data still delineate the effect of reopening an IRA on the circulating cytokine levels by head-to-head comparisons. Second, we did not have a third group with an occluded IRA which remained occluded after the coronary intervention to challenge the overall results achieved. However, with advanced techniques in a setting of an AMI, too few patients could be enrolled in that group. Last, serial changes in cytokine levels were not measured in this study although this could have provided more information. Since cytokine production varies widely following angioplasty procedures in individuals with different extents of myocardial injury, this study simply investigated whether opening an IRA can flush out significant amounts of cytokines. It, of course, would be interesting to assess whether there is a real "release curve" of these parameters by sequential sampling performed regularly for several days after recanalization. 
Conclusions: Our data raise the possibility that the benefits of late reperfusion of a totally occluded IRA in patients with ongoing myocardial ischemia may involve mechanisms associated with stem cell-mobilizing and plaque-stabilizing cytokines. This study also provides the rationale to investigate in the future serial changes in cytokines and the numbers of circulating stem/progenitor cells after reperfusion.

\section{REFERENCES}

1. Hochman JS, Lamas GA, Buller CE, et al. Coronary intervention for persistent occlusion after myocardial infarction. N Engl J Med 2006; 355: 2395-407.

2. Yousef ZR, Redwood SR, Bucknall CA, Sulke AN, Marber MS. Late intervention after anterior myocardial infarction: effects on left ventricular size, function, quality of life, and exercise tolerance: results of the Open Artery Trial (TOAT Study). J Am Coll Cardiol 2002; 40: 869-76.

3. Dzavík V, Buller CE, Lamas GA, et al. Randomized trial of percutaneous coronary intervention for subacute infarct-related coronary artery occlusion to achieve long-term patency and improve ventricular function: the Total Occlusion Study of Canada (TOSCA)-2 trial. Circulation 2006; 114: 2449-57.

4. Mangi AA, Noiseux N, Kong D, et al. Mesenchymal stem cells modified with Akt prevent remodeling and restore performance of infarcted hearts. Nat Med 2003; 9: 1195-201.

5. Kawamoto A, Gwon HC, Iwaguro H, et al. Therapeutic potential of ex vivo expanded endothelial progenitor cells for myocardial ischemia. Circulation 2001; 103: 634-7.

6. Kocher AA, Schuster MD, Szabolcs MJ, et al. Neovascularization of ischemic myocardium by human bone-marrow-derived angioblasts prevents cardiomyocyte apoptosis, reduces remodeling and improves cardiac function. Nat Med 2001; 7: 430-6.

7. Schächinger V, Erbs S, Elsässer A, et al. Intracoronary bone marrow-derived progenitor cells in acute myocardial infarction. N Engl J Med 2006; 355: 1210-21.

8. Orlic D, Kajstura J, Chimenti S, et al. Bone marrow cells regenerate infarcted myocardium. Nature 2001; 410: 701-5.

9. Ripa RS, Jørgensen E, Wang Y, et al. Stem cell mobilization induced by subcutaneous granulocytecolony stimulating factor to improve cardiac regeneration after acute ST-elevation myocardial infarction: result of the double-blind, randomized, placebo-controlled stem cells in myocardial infarction (STEMMI) trial. Circulation 2006; 113: 1983-92.

10. Zohlnhöfer D, Ott I, Mehilli J, et al. REVIVAL-2 Investigators. Stem cell mobilization by granulocyte colony-stimulating factor in patients with acute myocardial infarction: a randomized controlled trial. JAMA 2006; 295: 1003-10.

11. Ince H, Petzsch M, Kleine HD, et al. Prevention of left ventricular remodeling with granulocyte colony-stimulating factor after acute myocardial infarction: final 1-year results of the Front-Integrated Revascularization and Stem Cell Liberation in Evolving Acute Myocardial Infarction by Granulocyte Colony-Stimulating Factor (FIRSTLINE-AMI) Trial. Circulation 2005; 112: I73-80.

12. De Falco E, Porcelli D, Torella AR, et al. SDF-1 involvement in endothelial phenotype and ischemiainduced recruitment of bone marrow progenitor cells. Blood 2004; 104: 3472-82.

13. Powell TM, Paul JD, Hill JM, et al. Granulocyte colony-stimulating factor mobilizes functional endothelial progenitor cells in patients with coronary artery disease. Arterioscler Thromb Vasc Biol 2005; 25: 296-301.

14. Askari AT, Unzek S, Popovic ZB, et al. Effect of stromal-cell-derived factor 1 on stem-cell homing and tissue regeneration in ischaemic cardiomyopathy. Lancet 2003; 362: 697-703.

15. Urbanek K, Rota M, Cascapera S, et al. Cardiac stem cells possess growth factor-receptor systems that after activation regenerate the infarcted myocardium, improving ventricular function and long-term survival. Circ Res 2005; 97: 663-73.

16. Gill M, Dias S, Hattori K, et al. Vascular trauma induces rapid but transient mobilization of VEGFR2 (+) 
AC133 (+) endothelial precursor cells. Circ Res 2001; 88: 167-74.

17. Wojakowski W, Tendera M, Michałowska A, et al. Mobilization of CD34/CXCR4+, CD34/CD117+, c-met+ stem cells, and mononuclear cells expressing early cardiac, muscle, and endothelial markers into peripheral blood in patients with acute myocardial infarction. Circulation 2004; 110: 3213-20.

18. Zernecke A, Schober A, Bot I, et al. SDF-1alpha/CXCR4 axis is instrumental in neointimal hyperplasia and recruitment of smooth muscle progenitor cells. Circ Res 2005; 96: 784-91.

19. Frangogiannis NG, Perrard JL, Mendoza LH, et al. Stem cell factor induction is associated with mast cell accumulation after canine myocardial ischemia and reperfusion. Circulation 1998; 98: 687-98.

20. Suárez de Lezo J, Torres A, Herrera I, et al. Effects of stem-cell mobilization with recombinant human granulocyte colony stimulating factor in patients with percutaneously revascularized acute anterior myocardial infarction. Rev Esp Cardiol 2005; 58: 253-61. (Spanish)

21. Leone AM, Rutella S, Bonanno G, et al. Endogenous G-CSF and CD34+ cell mobilization after acute myocardial infarction. Int J Cardiol 2006; 111: 202-8.

22. Wojakowski W, Tendera M, Zebzda A, et al. Mobilization of CD34 (+), CD117 (+), CXCR4 (+), c-met $(+)$ stem cells is correlated with left ventricular ejection fraction and plasma NT-proBNP levels in patients with acute myocardial infarction. Eur Heart J 2006; 27: 283-9.

23. Yoshioka T, Takahashi M, Shiba Y, et al. Granulocyte colony-stimulating factor (G-CSF) accelerates reendothelialization and reduces neointimal formation after vascular injury in mice. Cardiovasc Res 2006; 70: 61-9.

24. Wang $\mathrm{CH}$, Verma S, Hsieh IC, et al. Enalapril increases ischemia-induced endothelial progenitor cell mobilization through manipulation of the CD26 system. J Mol Cell Cardiol 2006; 41: 34-43.

25. Abbott JD, Huang Y, Liu D, Hickey R, Krause DS, Giordano FJ. Stromal cell-derived factor-1alpha plays a critical role in stem cell recruitment to the heart after myocardial infarction but is not sufficient to induce homing in the absence of injury. Circulation 2004; 110: 3300-5.

26. Ma N, Stamm C, Kaminski A, et al. Human cord blood cells induce angiogenesis following myocardial infarction in NOD/scid-mice. Cardiovasc Res 2005; 66: 45-54.

27. Yamani MH, Ratliff NB, Cook DJ, et al. Peritransplant ischemic injury is associated with up-regulation of stromal cell-derived factor-1. J Am Coll Cardiol 2005; 46: 1029-35.

28. Damås JK, Waehre T, Yndestad A, et al. Stromal cell-derived factor-1alpha in unstable angina: potential antiinflammatory and matrix-stabilizing effects. Circulation 2002; 106: 36-42.

29. Tilton B, Ho L, Oberlin E, et al. Signal transduction by CXC chemokine receptor 4 Stromal cellderived factor 1 stimulates prolonged protein kinase B and extracellular signal-regulated kinase 2 activation in T lymphocytes. J Exp Med 2000; 192: 313-24.

30. Wang CH, Cherng WJ, Yang NI, et al. Cyclosporine increases ischemia-induced endothelial progenitor cell mobilization through manipulation of the CD26 system. Am J Physiol Regul Integr Comp Physiol 2008; 294: R811-8.

31. Fava RA, Olsen NJ, Spencer-Green G, et al. Vascular permeability factor/endothelial growth factor (VPF/VEGF): accumulation and expression in human synovial fluids and rheumatoid synovial tissue. J Exp Med 1994; 180: 341-6.

32. Ferrara N, Winer J, Burton T. Aortic smooth muscle cells express and secrete vascular endothelial growth factor. Growth Factors 1991; 5: 141-8

33. Broudy VC, Kovach NL, Bennett LG, Lin N, Jacobsen FW, Kidd PG. Human umbilical vein endothelial cells display high-affinity c-kit receptors and produce a soluble form of the c-kit receptor. Blood 1994; 83: 2145-52.

34. Grabarek J, Groopman JE, Lyles YR, et al. Human kit ligand (stem cell factor) modulates platelet activation in vitro. J Biol Chem 1994; 269: 21718-24. 